\title{
ABILITÀ METALINGUISTICHE NEGLI ALUNNI BILINGUI E MONOLINGUI
}

\author{
Andrea Močinić \\ Scuola elementare italiana di Buie \\ Neala Ambrosi-Randić \\ Dipartimento di studi in lingua italiana \\ Università Juraj Dobrila di Pola
}

\section{Riassunto}

La parte introduttiva di questo saggio presenta una rassegna di ricerche sul funzionamento cognitivo dei bilingui, nonché quelle riportanti i vantaggi e gli svantaggi del bilinguismo. Infatti, gli studi sul bilinguismo sono molti, ma i risultati ottenuti spesso non portano a conclusioni univoche. Per chiarire ed ampliare le conoscenze precedenti sul rapporto esistente tra bilinguismo e funzionamento metacognitivo, è stata condotta una ricerca su 230 alunni delle scuole elementari, bilingui e monolingui. I risultati ottenuti confermano l'effetto positivo dello sviluppo sulle abilità metacognitive ed una superiorità metalinguistica dei bambini monolingui, con una particolarità molto interessante discussa in seguito: i cambiamenti evolutivi sono molto più consistenti per i bambini bilingui.

Parole chiave: bilinguismo, metacognizione, bambini

\section{Introduzione}

Gli studi sul bilinguismo sono molteplici, e di conseguenza, molteplici sono anche le definizioni date. Deshays (2003) definisce il bilinguismo come la padronanza naturale di due lingue, e aggiunge che spesso si ritiene che una persona bilingue sia in grado di fondersi in due società che parlano lingue diverse senza farsi notare. Baker (2007), invece, descrive in modo un po' umoristico il concetto, affermando che da quando le biciclette hanno due ruote e il binocolo è fatto per guardarci con due occhi, sembrerebbe che il bilinguismo sia semplicemente parlare due lingue, però spiega subito che la faccenda non è poi così semplice.

Essere bilingue comporta dei vantaggi e svantaggi. Albers e Borgschulte (2009) individuano alcuni possibili campi in cui si possono raggruppare i vantaggi dell'essere 
bilingui. Tali campi comprendono vantaggi comunicativi (conoscenza di due lingue, possibilità di comunicare con un numero più grande di persone), vantaggi culturali (maggior ricchezza culturale, maggior livello di tolleranza), vantaggi cognitivi (maggior creatività e sensibilità nella comunicazione), vantaggi caratteriali (maggior fiducia in se stessi e nelle proprie possibilità), vantaggi scolastici (facilità maggiore nell'imparare una nuova lingua), fino ad arrivare ai vantaggi economici (maggiori possibilità d'impiego). Secondo Deshays (2003) ci sono anche dei vantaggi meno tangibili, come la comprensione, la tolleranza e l'apertura mentale nei confronti di altri popoli e di altri costumi, oppure l'arricchimento della personalità, che diventa il frutto di due culture, di due letterature, di due modi di vita.

Un altro campo nel quale sono stati trovati vantaggi a favore dei bambini bilingui è quello della sensibilità comunicativa. Si tratta della sensibilità dell'individuo in situazioni comunicative diverse, quando la persona deve adeguarsi all'ascoltatore nel comunicare con esso. Uno degli esperimenti più interessanti sul tema è stato effettuato da Genesee (1975; in Baker, 2007). L'esperimento comprendeva due gruppi di bambini, uno bilingue ed uno monolingue, che dovevano spiegare a dei loro compagni bendati le regole di un gioco, senza che i compagni bendati chiedessero loro ulteriori spiegazioni. I bambini bilingui spiegavano molto meglio le regole rispetto ai monolingui, e questi risultati dimostrano una maggior sensibilità in situazioni sociali che richiedono una comunicazione attenta: un bambino bilingue potrebbe essere più consapevole delle necessità dell'ascoltatore, cioè sembrerebbe che il suo livello empatico sia maggiore rispetto a quello del bambino monolingue.

Deshays (2003) scrive che un bambino che è esposto a due lingue fin dalla nascita le apprenderà entrambe in modo naturale nella loro interezza. L'autrice spiega che tutti i bambini sono potenzialmente capaci di apprendere qualsiasi lingua, essendo stato dimostrato che tutti gli esseri umani possiedono un'identica morfologia degli organi vocali. Inoltre Deshays (2003) parla di plasticità del cervello del bambino, un termine usato anche da Titone (1993), con il quale vuole spiegare che il cervello del bambino è ancora in via di sviluppo, è malleabile, assorbe informazioni dall'ambiente che lo circonda come fosse una spugna, a differenza del cervello di un adulto, il quale non ha più una tale capacità di raccolta di stimoli e informazioni. Infatti, tale flessibilità del cervello del bambino gli permette di apprendere con la stessa facilità una, due o tre lingue supplementari, come sostiene Baker (2007), secondo il quale sembra ci sia abbastanza spazio per imparare più di due lingue.

\subsection{Ricerche sul funzionamento cognitivo nei bilingui}

All'inizio del ventesimo secolo si aveva una visione generalmente negativa sulla questione del bilinguismo. Si sosteneva che un'educazione bilingue poteva portare alla comparsa di seri problemi psichici. Alcune ricerche, come ad esempio quella di Saer (1923; in Baker, 2007), affermavano che i bambini bilingui generalmente risolvono peggio i test d'intelligenza dei loro compagni monolingui. Si è poi dimostrato che tali studi avevano serie mancanze metodologiche, e i risultati, quindi, non potevano venir presi in considerazione. Si trattava del primo periodo di studi sugli effetti del bilinguismo 
sull'intelligenza verbale, il periodo degli effetti dannosi (Baker, 2007). Nel secondo periodo degli effetti neutrali, gli studi effettuati non riportavano differenze tra l'intelligenza di bilingui e monolingui. Mentre il terzo ed ultimo, chiamato periodo degli effetti additivi, riporta risultati migliori da parte di individui bilingui rispetto a quelli monolingui.

La prima ricerca che riportò aspetti positivi del bilinguismo fu quella di Peal e Lambert (1962; in Baker, 2007). La teoria da loro concepita venne chiamata ipotesi dello scambio-codici. Le loro conclusioni furono che il bilinguismo porta ad una maggiore flessibilità mentale; l'abilità di pensare meglio in modo astratto, indipendentemente da parole, alla superiorità nella formazione di concetti, e altri benefici per l'intelligenza dei bilingui.

Analizzando il tema della relazione tra cognitivismo e bilinguismo, Johnson (2003) riporta alcune classi di compiti verbali nelle quali i bilingui dimostrano dei vantaggi rispetto ai loro compagni monolingui. Il primo viene chiamato gruppo dei compiti verbali in situazioni ingannevoli, e si tratta di compiti che creano nei soggetti dei conflitti riguardanti le soluzioni possibili, legate a due tipi di struttura diversa. Un esempio riportato dall'autrice riguarda il giudicare se certe frasi date sono grammaticalmente corrette o meno. Il secondo gruppo viene chiamato dei compiti percettivo-cognitivi in situazioni ingannevoli, nei quali l'organizzazione iniziale dello stimolo cambia e diventa ingannevole, chiedendo all'individuo una riorganizzazione dello stimolo. Un esempio di quest'ultimo tipo di compiti può essere quello classico della conservazione dei liquidi (bicchieri di diverse dimensioni con uguale quantità di liquido). Il terzo gruppo di compiti dove i bilingui dimostrano vantaggi, infine, viene chiamato dei compiti richiedent $i$ controllo linguistico di azioni palesi in situazioni ingannevoli. Si tratta di compiti che riguardano le abilità linguistiche nell'iniziare un'azione da parte del bambino, il quale deve poi essere in grado di mantenere attivo il termine linguistico durante un periodo di indugio.

\subsection{Ricerche sulle abilità metalinguistiche nei bilingui}

Molti autori discutono della consapevolezza metalinguistica nei loro lavori, dandone anche diverse definizioni. Così Malakoff e Hakuta (2003) affermano che essa sia la consapevolezza della basilare natura linguistica nell'uso della lingua, spiegando che tale consapevolezza aiuta l'individuo a staccarsi dalla comprensione o dalla produzione di una parola per poter considerare la forma linguistica e la struttura fondamentale del significato della parola. Diaz e Klingler (2003), invece, spiegano che si tratta di un certo numero di abilità coinvolgenti una consapevolezza oggettiva e un controllo di variabili linguistiche. Un compito metalinguistico, invece, è il pensare alla natura linguistica del messaggio: curare e riflettere sugli aspetti strutturali della lingua.

Varie ricerche hanno riportato effetti positivi del bilinguismo in alcune abilità metalinguistiche, tra le quali: distinzione prima parola - referente (Ianco-Worrall, 1972; in Diaz e Klingler, 2003), sensibilità verso la struttura linguistica e i dettagli (Ben-Zeev, 1977; in Diaz e Klingler, 2003), scoperta di ambiguità e analisi di frasi tautologiche (Cummins e Mulchany, 1978; in Diaz e Klingler, 2003), orientamento sintattico nell'elaborazione della frase (Galambos, 1985; in Diaz e Klingler, 2003), correzione di frasi 
non grammaticali e scoperta di mescolamento linguistico (Diaz, 1985; in Diaz e Klingler, 2003), controllo dell'elaborazione linguistica (Bialystok, 1986; in Diaz e Klingler, 2003), comprensione dell'arbitrarietà referenziale e non sincretica del linguaggio (Edwards e Christophersen, 1988; in Diaz e Klingler, 2003).

L'idea di una dominanza metalinguistica dei bilingui sui monolingui è partita dalle idee di Vygotsky (1962; in Diaz e Klingler, 2003), il quale credeva che i bilingui, vedendo la loro lingua come un sistema tra i tanti, godevano di una speciale consapevolezza e controllo delle proprie operazioni linguistiche. Baker (2007) riporta l'esempio della ricerca di Doyle (1978), il quale scrive che i bilingui tendono ad essere superiori nell'abilità di mettere in relazione storie ed esprimere concetti all'interno di queste storie quando comparati con i monolingui. Successivi studi, come ad esempio Ianco-Worrall (1972; in Baker, 2007), Ben-Zeev (1977; in Baker, 2007), e altri, hanno portato alla conclusione che mentre i monolingui tendono ad essere limitati dalle parole, i bilingui tendono a credere che la lingua sia più arbitraria. Baker (2007) aggiunge che per i bilingui, nomi e oggetti sono separati. Infatti, conoscendo due diverse lingue e due diversi modi per scrivere le stesse parole, i bambini bilingui sono capaci di capire queste rappresentazioni simboliche prima dei loro coetanei monolingui. Infine, si potrebbe concludere che i vantaggi dei bambini bilingui sui loro coetanei monolingui legati alle capacità metalinguistiche, dipendono probabilmente dalla necessità e dall'abitudine dei bilingui di fare differenza fra le loro due lingue.

In conclusione, possiamo dire che la maggior parte delle ricerche ha riportato risultati positivi, stando a significare che un'educazione bilingue può essere vantaggiosa per il bambino, ma anche per l'adulto. Ci sono diversi effetti positivi, sia nel campo linguistico, che in quello psicologico; essi si estendono dal campo lavorativo a quello empatico della persona, toccano anche la personalità, il carattere, il modo di vedere il mondo e di confrontarsi della persona con le altre culture. La precocità dell'educazione al bilinguismo è di primaria importanza, considerata la plasticità del cervello del bambino che lo aiuta ad apprendere le lingue nel modo migliore.

In seguito a questo inquadramento teorico sul bilinguismo, si delinea la necessità di discutere i problemi che si trovano alla base di questa ricerca. Le domande a cui si vuole rispondere riguardano il funzionamento cognitivo nei bambini monolingui e bilingui, e soprattutto la capacità di risolvere compiti metalinguistici. Gli obiettivi di conseguenza sono due: verificare i cambiamenti evolutivi nella capacità di risolvere compiti metalinguistici; e verificare l'esistenza di differenze nella capacità di risolvere compiti metalinguistici tra bambini monolingui e bilingui.

\section{Metodologia della ricerca}

\subsection{Partecipanti}

Alla ricerca hanno partecipato 230 alunni (107 maschi e 123 femmine), delle seconde e quarte classi di quattro scuole elementari: due con insegnamento in lingua italiana (Pola 85 e Buie 16 alunni), e due con insegnamento in lingua croata (Pola 89 e Buie 40). 
Per verificare i cambiamenti evolutivi, come campioni di ricerca sono stati scelti gli alunni delle seconde e delle quarte classi, dell'età media di 8 nel primo e rispettivamente 10 anni nel secondo campione.

Gli alunni frequentanti le scuole in lingua italiana sono stati classificati come bilingui, prendendo in considerazione il fatto che tutte le lezioni vengono fatte in lingua italiana, cioè la lingua della minoranza, oltre alle lezioni di lingua croata. Gli alunni delle scuole in lingua croata, invece, sono stati classificati come bambini monolingui ${ }^{[1]}$ perché, a differenza degli alunni delle scuole in lingua italiana, hanno un numero di ore settimanali ed un programma di L2 meno consistenti ${ }^{[2]}$.

\subsection{Procedimento}

La ricerca è stata compiuta nelle scuole partecipanti, nel corso delle lezioni regolari, con la durata di 15-20 minuti per classe, da marzo a maggio del 2009. In classe, il ricercatore spiegava agli alunni cosa avrebbero dovuto fare, presentando due questionari $^{[3]}$ con delle domande a scelta multipla. Il ricercatore assicurava gli alunni che non c'erano risposte giuste o sbagliate e che non avrebbero ricevuto nessun voto per il lavoro effettuato. Le domande venivano lette agli alunni, e poi veniva loro lasciato il tempo necessario per pensare e segnare la risposta.

\subsection{Strumenti}

Come punto di partenza per la costruzione del questionario sono serviti vari test di ricercatori che hanno in precedenza affrontato argomenti simili. Il questionario usato ha due versioni diverse: italiana per gli alunni delle scuole con insegnamento in lingua italiana, e croata per gli alunni delle scuole con insegnamento in lingua croata. Il questionario mirava a misurare le abilità metalinguistiche nei bambini, e più specificamente la capacità di capire le relazioni convenzionali tra le parole ed i significati ad esse attribuiti. I compiti proposti richiedono al bambino la sostituzione di simboli. Un esempio di compito con la sostituzione di simboli è quello effettuato da Ben-Zeev (1977; in Bialystok, 2006), nel quale si chiedeva ai bambini di usare al posto del pronome noi la parola spaghetti, costruendo poi frasi con lo scambio di parole (es. Spaghetti sono bravi bambini.).

Il questionario è stato costruito sulla base della famosissima ricerca sul problema oramai classico, del Sole e della Luna di Piaget (1929; in Bialystok, 2006). Il testo ${ }^{[4]}$ introduttivo consiste nel gioco dello scambio di nomi del Sole e della Luna. Seguono sei domande (figurano nella tabella 1) che cercano di confondere il lettore e provare le sue capacità metalinguistiche e logiche. Le risposte a disposizione sono tre per ogni

\footnotetext{
[1] Anche se al giorno d’oggi è quasi impossibile trovare dei perfetti monolingui, riteniamo che i bambini che hanno appena iniziato con lapprendimento di una L2 (nella $2^{\mathrm{a}}$ classe) o lo fanno da 2 anni (nella $4^{\mathrm{a}}$ classe) non sono ancora in grado di usare un'altra lingua accanto a quella materna, e quindi per il momento si possono ancora definire prevalentemente monolingui.

[2] Le scuole in lingua italiana hanno 5 ore settimanali di lingua croata e 2 ore settimanali di lingua inglese, mentre le scuole in lingua croata hanno 3 ore settimanali della prima lingua straniera e 2 ore settimanali della seconda lingua straniera.

[3] La ricerca fa parte della tesi di laurea Bilinguismo e abilità metacognitive (Močinić, 2010) e in questa sede riportiamo soltanto una parte dei dati ottenuti.

[4] Immaginate che la Luna ed il Sole si scambino i nomi: il Sole si chiamerebbe Luna e viceversa, la Luna si chiamerebbe Sole. Cercate ora di immaginare la storia e rispondete alle domande.
} 
domanda: nelle prime quattro le risposte possibili sono Sole, Luna e non so; mentre nella quinta e nella sesta sono nero, chiaro e non so.

\section{Risultati della ricerca}

Per rispondere ai due problemi principali della ricerca, abbiamo analizzato le risposte degli alunni ed effettuato una serie di analisi statistiche che riportiamo in seguito.

\subsection{Differenze evolutive nelle abilità metalinguistiche}

Per esplorare l'eventuale esistenza di differenze evolutive abbiamo analizzato le risposte date dagli alunni nelle seconde e quarte classi. Dai risultati presentati nella Tabella 1 è possibile vedere l'effetto dell'età nelle abilità metacognitive dei bambini. In quattro su sei situazioni proposte i bambini più grandi ( $4^{\mathrm{a}}$ classe) risolvono in un numero significativamente maggiore in modo corretto i compiti rispetto a quelli più piccoli.

Tabella 1. Frequenze e percentuali delle risposte alle domande del Questionario La storiella del Sole e della Luna date dai bambini della $2^{a}$ e $4^{a}$ classe

\begin{tabular}{|c|c|c|c|c|}
\hline Domande & Classe & $\begin{array}{c}\text { Risposte } \\
\text { esatte }\end{array}$ & $\begin{array}{l}\text { Risposte } \\
\text { sbagliate }\end{array}$ & $\begin{array}{c}\text { Chi quadrato } \\
\text { df }=1\end{array}$ \\
\hline $\begin{array}{l}\text { 1. Uno dei due sorge al } \\
\text { mattino. Quale sarebbe il suo } \\
\text { nome?* }\end{array}$ & II & $\begin{array}{c}39 \\
39,8 \% \\
105 \\
90,5 \%\end{array}$ & $\begin{array}{c}58 \\
59,2 \% \\
11 \\
9,5 \%\end{array}$ & $\begin{array}{l}58,78 \\
\mathrm{p}<.001\end{array}$ \\
\hline 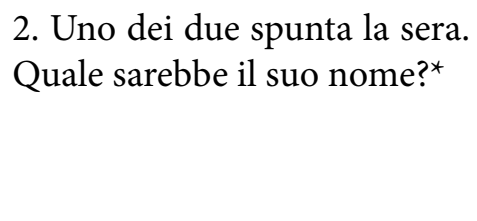 & IV & $\begin{array}{c}38 \\
38,8 \% \\
105 \\
90,5 \%\end{array}$ & $\begin{array}{c}59 \\
60,2 \% \\
11 \\
9,5 \%\end{array}$ & $\begin{array}{c}60,81 \\
\mathrm{p}<.001\end{array}$ \\
\hline $\begin{array}{l}\text { 3. Chi starebbe nel cielo } \\
\text { durante il giorno?* }\end{array}$ & IV & $\begin{array}{c}39 \\
39,8 \% \\
106 \\
91,4 \%\end{array}$ & $\begin{array}{c}58 \\
59,2 \% \\
9 \\
7,8 \%\end{array}$ & $\begin{array}{l}63,36 \\
\mathrm{p}<.001\end{array}$ \\
\hline $\begin{array}{l}\text { 4. Chi starebbe nel cielo } \\
\text { durante la sera?* }\end{array}$ & IV & $\begin{array}{c}38 \\
38,8 \% \\
105 \\
90,5 \%\end{array}$ & $\begin{array}{c}60 \\
61,2 \% \\
10 \\
8,6 \%\end{array}$ & $\begin{array}{c}63,81 \\
\mathrm{p}<.001\end{array}$ \\
\hline
\end{tabular}




\begin{tabular}{|l|c|c|c|c|}
\hline $\begin{array}{l}\text { 5. Di che colore sarebbe il } \\
\text { cielo di notte? }\end{array}$ & II & $\begin{array}{c}59 \\
60,2 \%\end{array}$ & $\begin{array}{c}39 \\
39,8 \%\end{array}$ & \\
& IV & $\begin{array}{c}55 \\
47,4 \%\end{array}$ & $\begin{array}{c}61 \\
52,6 \%\end{array}$ & $\mathrm{p}=\mathrm{n} . \mathrm{s}$. \\
\hline $\begin{array}{l}\text { 6. Di che colore sarebbe il } \\
\text { cielo di giorno? }\end{array}$ & II & $\begin{array}{c}60 \\
61,2 \%\end{array}$ & $\begin{array}{c}38 \\
38,8 \%\end{array}$ & \\
& IV & $\begin{array}{c}56 \\
48,3 \%\end{array}$ & $\begin{array}{c}60 \\
51,7 \%\end{array}$ & $\begin{array}{c}\text { p }=\text { n.s. } \\
\end{array}$ \\
\hline
\end{tabular}

* - uno o due alunni hanno scelto la risposta "non so"

\subsection{Differenze nelle abilità metalinguistiche tra bambini bilingui e} monolingui

Il secondo passo dell'analisi è stato compiuto con l'intenzione di verificare l'eventuale esistenza di differenze nelle abilità metalinguistiche tra alunni bilingui e monolingui. I risultati presentati nella Tabella 2 parlano a favore dei bambini monolingui. Infatti, in 4 compiti proposti su 6 sono appunto i bambini monolingui quelli che risolvono i problemi con maggior successo.

Tabella 2. Frequenze e percentuali delle risposte dei bilingui e monolingui alle domande del Questionario

\begin{tabular}{|c|c|c|c|c|}
\hline Domande & $\begin{array}{l}\text { Bilingui } \\
\text { Monolingui }\end{array}$ & $\begin{array}{l}\text { Risposte } \\
\text { esatte }\end{array}$ & $\begin{array}{l}\text { Risposte } \\
\text { sbagliate }\end{array}$ & $\begin{array}{c}\text { Chi } \\
\text { quadrato } \\
\text { df }=1\end{array}$ \\
\hline $\begin{array}{l}\text { 1. Uno dei due sorge al } \\
\text { mattino. Quale sarebbe il suo } \\
\text { nome?* }\end{array}$ & M & $\begin{array}{c}52 \\
51,5 \% \\
92 \\
81,4 \% \\
\end{array}$ & $\begin{array}{c}48 \\
47,5 \% \\
\\
21 \\
18,6 \% \\
\end{array}$ & $\begin{array}{c}19,64 \\
\mathrm{p}<.001\end{array}$ \\
\hline $\begin{array}{l}\text { 2. Uno dei due spunta la sera. } \\
\text { Quale sarebbe il suo nome?* }\end{array}$ & M & $\begin{array}{c}51 \\
50,5 \% \\
92 \\
81,4 \% \\
\end{array}$ & $\begin{array}{c}49 \\
48,5 \% \\
21 \\
(18,6 \%)\end{array}$ & $\begin{array}{l}20,89 \\
\mathrm{p}<.001\end{array}$ \\
\hline $\begin{array}{l}\text { 3. Chi starebbe nel cielo } \\
\text { durante il giorno?* }\end{array}$ & M & $\begin{array}{c}52 \\
51,5 \% \\
93 \\
82,3 \% \\
\end{array}$ & $\begin{array}{c}47 \\
46,5 \% \\
\\
20 \\
17,7 \% \\
\end{array}$ & $\begin{array}{c}20,29 \\
\mathrm{p}<.001\end{array}$ \\
\hline
\end{tabular}




\begin{tabular}{|l|c|c|c|c|}
\hline $\begin{array}{l}\text { 4. Chi starebbe nel cielo } \\
\text { durante la sera?* }\end{array}$ & $\mathrm{B}$ & $\begin{array}{c}53 \\
54,5 \%\end{array}$ & $\begin{array}{c}47 \\
46,5 \%\end{array}$ & 15,89 \\
& $\mathrm{M}$ & $\begin{array}{c}90 \\
79,6 \%\end{array}$ & $\begin{array}{c}23 \\
20,4 \%\end{array}$ & $\mathrm{p}<.001$ \\
\hline $\begin{array}{l}\text { 5. Di che colore sarebbe il } \\
\text { cielo di notte? }\end{array}$ & $\mathrm{B}$ & 55 & 46 & \\
& & $54,5 \%$ & $45,5 \%$ & \\
& $\mathrm{M}$ & 59 & 54 & $\mathrm{p}=\mathrm{n} . \mathrm{s}$. \\
\hline 6. Di che colore sarebbe il & $\mathrm{B}$ & $52,2 \%$ & $47,8 \%$ & \\
cielo di giorno? & & $55,4 \%$ & $44,6 \%$ & \\
& $\mathrm{M}$ & 60 & 53 & $\mathrm{p}=\mathrm{n} . \mathrm{s}$. \\
\hline
\end{tabular}

* - uno o 2 alunni hanno scelto la risposta "non so"

Visto che abbiamo riscontrato delle differenze evolutive come pure quelle inerenti lo status linguistico, di conseguenza ci siamo chiesti se lo sviluppo delle abilità metacognitive procede a pari passo per gli alunni bilingui e monolingui oppure ci sono delle differenze. Nella Tabella 3 figurano i risultati dei bambini bilingui e monolingui delle seconde classi mentre nella Tabella 4 i risultati dei bambini bilingui e monolingui delle quarte. Per quanto riguarda i bambini più piccoli $\left(2^{\mathrm{a}}\right.$ classe) i risultati sono molto sorprendenti: in 4 situazioni su 6 sono i monolingui ad essere migliori, mentre in due sono significativamente migliori i bilingui. Quello che succede nei bambini più grandi rappresenta sicuramente una delle sorprese maggiori di questa ricerca: nella quarta classe sono nuovamente i bambini monolingui a dimostrare una netta superiorità nelle capacità metacognitive in quanto risolvono meglio 5 compiti su 6.

Tabella 3. Differenze nelle abilità metalinguistiche tra bambini bilingui e monolingui delle classi seconde

\begin{tabular}{|c|c|c|c|c|}
\hline Domande & $\begin{array}{c}\text { Bilingui } \\
\text { Monolingui }\end{array}$ & $\begin{array}{l}\text { Risposte } \\
\text { esatte }\end{array}$ & $\begin{array}{l}\text { Risposte } \\
\text { sbagliate }\end{array}$ & $\begin{array}{c}\text { Chi quadrato } \\
\text { df }=1\end{array}$ \\
\hline $\begin{array}{l}\text { 1. Uno dei due sorge al } \\
\text { mattino. Quale sarebbe il } \\
\text { suo nome?* }\end{array}$ & B & $\begin{array}{c}16 \\
28,6 \% \\
23 \\
54,8 \% \\
\end{array}$ & $\begin{array}{c}39 \\
69,6 \% \\
19 \\
45,2 \% \\
\end{array}$ & $\begin{array}{l}5,50 \\
p<.05\end{array}$ \\
\hline $\begin{array}{l}\text { 2. Uno dei due spunta la sera. } \\
\text { Quale sarebbe il suo nome?* }\end{array}$ & M & $\begin{array}{c}15 \\
26,8 \% \\
\\
23 \\
54,8 \% \\
\end{array}$ & $\begin{array}{c}40 \\
71,4 \% \\
19 \\
45,2 \% \\
\end{array}$ & $\begin{array}{c}6,44 \\
p<.05\end{array}$ \\
\hline
\end{tabular}




\begin{tabular}{|c|c|c|c|c|}
\hline $\begin{array}{l}\text { 3. Chi starebbe nel cielo } \\
\text { durante il giorno?* }\end{array}$ & B & $\begin{array}{c}15 \\
26,8 \% \\
\\
24 \\
57,1 \% \\
\end{array}$ & $\begin{array}{c}40 \\
71,4 \% \\
18 \\
42,9 \% \\
\end{array}$ & $\begin{array}{c}7,64 \\
\mathrm{p}<.001\end{array}$ \\
\hline $\begin{array}{l}\text { 4. Chi starebbe nel cielo } \\
\text { durante la sera? }\end{array}$ & B & $\begin{array}{c}16 \\
28,6 \% \\
22 \\
52,4 \%\end{array}$ & $\begin{array}{c}40 \\
71,4 \% \\
20 \\
47,6 \%\end{array}$ & $\begin{array}{c}4,77 \\
\mathrm{p}<.05\end{array}$ \\
\hline $\begin{array}{l}\text { 5. Di che colore sarebbe il } \\
\text { cielo di notte? }\end{array}$ & $\mathrm{M}$ & $\begin{array}{c}44 \\
78,6 \% \\
\\
15 \\
35,7 \% \\
\end{array}$ & $\begin{array}{c}12 \\
21,4 \% \\
27 \\
64,3 \% \\
\end{array}$ & $\begin{array}{c}16,65 \\
p<.001\end{array}$ \\
\hline $\begin{array}{l}\text { 6. Di che colore sarebbe il } \\
\text { cielo di giorno? }\end{array}$ & $\mathrm{M}$ & $\begin{array}{c}45 \\
80,4 \% \\
15 \\
35,7 \%\end{array}$ & $\begin{array}{c}11 \\
19,6 \% \\
27 \\
64,3 \%\end{array}$ & $\begin{array}{c}18,31 \\
\mathrm{p}<.001\end{array}$ \\
\hline
\end{tabular}

* - un alunno ha scelto la risposta "non so"

Tabella 4. Differenze nelle abilità metalinguistiche tra bambini bilingui e monolingui delle classi quarte

\begin{tabular}{|c|c|c|c|c|}
\hline Domande & $\begin{array}{c}\text { Bilingui } \\
\text { Monolingui }\end{array}$ & $\begin{array}{c}\text { Risposte } \\
\text { esatte }\end{array}$ & $\begin{array}{l}\text { Risposte } \\
\text { sbagliate }\end{array}$ & $\begin{array}{c}\text { Chi quadrato } \\
\mathbf{d f}=1\end{array}$ \\
\hline $\begin{array}{l}\text { 1. Uno dei due sorge al } \\
\text { mattino. Quale sarebbe il suo } \\
\text { nome? }\end{array}$ & $\begin{array}{l}\mathrm{B} \\
\mathrm{M}\end{array}$ & $\begin{array}{c}36 \\
80 \% \\
\\
69 \\
97,2 \%\end{array}$ & $\begin{array}{c}9 \\
20 \% \\
2 \\
2,8 \%\end{array}$ & $\begin{array}{l}7,58 \\
\mathrm{p}<.01\end{array}$ \\
\hline $\begin{array}{l}\text { 2. Uno dei due spunta la sera. } \\
\text { Quale sarebbe il suo nome? }\end{array}$ & B & $\begin{array}{c}36 \\
80 \% \\
69 \\
97,2 \% \\
\end{array}$ & $\begin{array}{c}9 \\
20 \% \\
2 \\
2,8 \% \\
\end{array}$ & $\begin{array}{c}7,58 \\
\mathrm{p}<.01\end{array}$ \\
\hline $\begin{array}{l}\text { 3. Chi starebbe nel cielo } \\
\text { durante il giorno?* }\end{array}$ & B & $\begin{array}{c}37 \\
82,2 \% \\
69 \\
97,2 \%\end{array}$ & $\begin{array}{c}7 \\
15,6 \% \\
2 \\
2,8 \%\end{array}$ & $\begin{array}{c}4,77 \\
\mathrm{p}<.05\end{array}$ \\
\hline
\end{tabular}




\begin{tabular}{|c|c|c|c|c|}
\hline $\begin{array}{l}\text { 4. Chi starebbe nel cielo } \\
\text { durante la sera?* }\end{array}$ & $\mathrm{M}$ & $\begin{array}{c}37 \\
82,2 \% \\
68 \\
95,8 \% \\
\end{array}$ & $\begin{array}{c}7 \\
15,6 \% \\
3 \\
4,2 \% \\
\end{array}$ & $\begin{array}{c}3,31 \\
\mathrm{p}=\text { n.s. }\end{array}$ \\
\hline $\begin{array}{l}\text { 5. Di che colore sarebbe il } \\
\text { cielo di notte? }\end{array}$ & $\mathrm{M}$ & $\begin{array}{c}11 \\
24,4 \% \\
44 \\
62 \% \\
\end{array}$ & $\begin{array}{c}34 \\
75,6 \% \\
27 \\
38 \% \\
\end{array}$ & $\begin{array}{l}14,09 \\
\mathrm{p}<.01\end{array}$ \\
\hline $\begin{array}{l}\text { 6. Di che colore sarebbe il } \\
\text { cielo di giorno? }\end{array}$ & $\mathrm{M}$ & $\begin{array}{c}11 \\
24,4 \% \\
45 \\
63,4 \%\end{array}$ & $\begin{array}{c}34 \\
75,6 \% \\
26 \\
36,6 \%\end{array}$ & $\begin{array}{l}15,20 \\
\mathrm{p}<.01\end{array}$ \\
\hline
\end{tabular}

* - un alunno ha scelto la risposta "non so"

\section{Discussione dei risultati}

Fino ad oggi sono state compiute molte ricerche sull'influsso positivo o negativo del bilinguismo sullo sviluppo cognitivo di un bambino. I risultati ottenuti non danno risposte univoche: ci sono studi che riportano effetti negativi del bilinguismo sullo sviluppo cognitivo dell'individuo, come quelli di Nelson (1985; in Scotti Jurić, 2003), Mac Namara (1966; in Scotti Jurić, 2003) o Rosenblum e Pinker (1983; in Bialystok, 2006); e poi ci sono altri che confermano migliori abilità linguistiche e cognitive dei bilingui, come quelli di McLaughlin (1984; in Scotti Jurić, 2003), Peal e Lambert (1962; in Scotti Jurić, 2003), Ricciardelli (1992; in Bialystok, 2006) o Cummins (1984; in Scotti Jurić, 2003). Le ricerche citate sono state eseguite in paesi anglofoni, prevalentemente negli Stati Uniti. Siccome simili studi non sono mai stati compiuti per i bilingui d'Istria, si è deciso di vedere quali sarebbero stati i risultati in quest'area geografica.

Per quanto riguarda lo sviluppo, il questionario usato nella presente ricerca ha dimostrato chiaramente che con l'avanzare dell'età in generale i bambini risolvono meglio i compiti metalinguistici assegnati. Nel questionario La storiella del Sole e della Luna, i risultati sono a favore degli alunni delle quarte classi con un rapporto di quattro risposte esatte su sei. Possiamo concludere che i dati ottenuti indicano l'esistenza di effetti positivi dello sviluppo sulle abilità metalinguistiche.

Parlando di bilingui e monolingui, i risultati rilevati da questa ricerca non coincidono con quelli ottenuti dalla maggior parte degli studi consultati, come ad esempio Ianco-Worall (1972; in Baker, 2007), Ben-Zeev (1977; in Baker, 2007), Bialystok (1987; in Baker, 2007), Cummins (1978; in Bialystok, 2006), Ricciardelli (1992; in Bialystok, 2006). Infatti, le risposte al nostro questionario sono a favore dei bambini monolingui. Vedendo i risultati ottenuti, si è voluto poi analizzare i dati più a fondo, controllando le differenze tra gli alunni monolingui e bilingui delle classi seconde, e 
quelli delle quarte. Tale analisi ha confermato i risultati precedenti, rilevando però delle particolarità inattese. Gli alunni monolingui delle seconde classi hanno risposto meglio alle domande del questionario: i bambini monolingui hanno quattro risposte esatte su sei a favore, mentre le restanti due domande sono a favore dei loro coetanei bilingui. Il fatto che gli alunni bilingui abbiano risposto in modo esatto in una percentuale assai più alta dei loro coetanei monolingui alla quinta e alla sesta domanda del questionario $\mathrm{La}$ storiella del Sole e della Luna, sembra molto interessante e difficile da spiegare in quanto si tratta di due compiti che generalmente risultano più difficili da risolvere. Come mai ci sono riusciti i bilingui più piccoli? Specie in quanto poi nella quarta classe soltanto un numero ridotto di bilingui riesce a farlo? Una spiegazione si può ricercare nel fatto che il bilinguismo rallenta l'acquisizione di alcune abilità metalinguistiche, come riporta Hakuta (1987; in Diaz e Klingler, 2003).

Mettendo in seguito a confronto poi le tabelle con i risultati degli alunni delle seconde e delle quarte bilingui e dei loro coetanei monolingui, abbiamo notato un altro fatto molto interessante: $\mathrm{i}$ cambiamenti evolutivi sono molto più consistenti per i bambini bilingui. In due anni l'aumento è diverso: si passa dal $23-27 \%$ all' $80-82 \%$ di risposte esatte per i bilingui, il che corrisponde al $53 \%$ in più, mentre nello stesso periodo i monolingui passano dal 52-56-57\% al 97\% di risposte corrette, il che corrisponde ad un aumento del 30-35\%. Questi dati dimostrano un salto evolutivo molto più grande nei bilingui rispetto ai monolingui. Inoltre, mentre i monolingui continuano in modo lineare l'acquisizione nel campo delle abilità metalinguistiche, per i bilingui si presenta un fenomeno inverso. Le risposte esatte alle prime quattro domande sono a favore degli alunni delle quarte (27$28-29 \%$ verso $80-82 \%$ ), mentre le risposte esatte alla quinta e alla sesta domanda sono a favore degli alunni delle seconde (79-80\% a confronto con $24 \%$ ), con una differenza che varia tra il $52 \%$ e $56 \%$. Questi dati fanno pensare che il bilinguismo, in certi periodi, rallenta lo sviluppo delle abilità metalinguistiche, come riportato da Hakuta (1987; in Diaz e Klingler, 2003), ma poi in un' età più adulta può rappresentare un vantaggio per la persona, come suggerito da De Bot e Makoni (2005; in Baker, 2007).

Gli alunni bilingui delle quarte invece, hanno avuto risultati inferiori rispetto ai loro compagni monolingui. I risultati ottenuti dal questionario La storiella del Sole e della Luna parlano assolutamente in merito dei bambini monolingui: cinque risposte esatte su sei a loro favore. Bialystok (2006), ma soprattutto Baker (2007), scrivono che i risultati migliori dimostrati da bambini bilingui nei confronti dei loro coetanei monolingui nel risolvere compiti metalinguistici, dipendono molto dal livello di bilinguismo che un individuo possiede. Infatti, tali autori hanno riscontrato risultati positivi per bambini bilingui soltanto nel caso di un bilinguismo equilibrato, di equilingui. Se invece un bambino non possiede capacità linguistiche uguali o simili nelle due lingue, il suo bilinguismo non gli porta vantaggi cognitivi o metalinguistici. Qui ci possiamo collegare alla teoria delle soglie, secondo la quale i bilingui devono raggiungere una certa soglia o livello di competenze linguistiche in entrambe le lingue per riuscire ad avere determinati vantaggi a livello metalinguistico e cognitivo in generale. I bambini coinvolti dalla presente ricerca probabilmente non possiedono un simile livello di bilinguismo, o almeno la gran parte di essi, e di conseguenza non possono usufruire dei vantaggi che esso comporta. Visto che non siamo certi del livello di bilinguismo dei bambini 
intervistati, questo rimane un compito invitante per le ricerche in futuro.

$\mathrm{Si}$ potrebbe concludere che $\mathrm{i}$ risultati ottenuti in questa ricerca sono prevalentemente, ma non completamente, a favore degli alunni monolingui, a causa di un mancato equilinguismo nella maggior parte dei bambini delle scuole italiane dell'Istria. Inoltre, bisogna considerare il fatto che sono stati presi dalla ricerca soltanto i bambini della seconda e quarta classe elementare (rispettivamente di 8 e 10 anni), mentre nelle ricerche che hanno trovato effetti positivi per i bilingui, come quelle di Ianco-Worrall (1972; in Baker, 2007), ad esempio, tra i bambini intervistati erano inclusi anche quelli prescolari (da 4 a 6 anni), ed altrettanto in Ben-Zeev (1977; in Bialystok, 2006). I nostri risultati sembrano confermare quelli di Hakuta (1987; in Diaz e Klingler, 2003). Si tratta delle conclusioni tratte dai risultati di una sua ricerca fatta su bambini di età diverse, dalla scuola materna alla sesta classe della scuola elementare. Secondo questa ricerca ci sono degli effetti positivi del bilinguismo sul funzionamento cognitivo dei bambini della scuola materna e della prima classe elementare, mentre i vantaggi si attenuano con il passare degli anni. Infatti, dalla quarta alla sesta i bambini bilingui dimostrano sempre meno vantaggi cognitivi rispetto ai loro compagni monolingui, per finire con nessun effetto positivo significante nelle classi quinte e seste.

Riteniamo che in seguito sarebbe molto utile ed interessante approfondire questo studio allargando il campione di ricerca, prendendo in considerazione nuove variabili, quali il livello di conoscenza dell'italiano e del croato, la lingua parlata con la madre, con il padre, la lingua maggiormente usata nel circondario del bambino. Questo permetterebbe di rispondere a molte domande: come influiscono sul bambino i genitori che parlano lingue diverse, se sia possibile migliorare a scuola il livello di bilinguismo dei bambini, o come influisce il bilinguismo sul funzionamento emotivo, cognitivo e sociale del bambino nel corso dello sviluppo.

\section{BIBLIOGRAFIA}

ALBERS, K., BORGSCHULTE, U., 2009, Bilingualism in the family; in: http://egora.uni-muenster.de/ew/persoenlich/scheerer/.../12-in-the-famliy.doc; 6.7.2009 BAKER, C., 2007, Foundation of bilingual education and bilingualism, Multilingual Matters LTD, Bristol.

BIALYSTOK, E., 2006, Bilingualism in development: language, literacy \& cognition, Cambridge University Press, Cambridge.

DESHAYS, E., 2003, Come favorire il bilinguismo dei bambini, Red edizioni, Novara.

DIAZ, R. M., KLINGLER, C., 2003, Language processing in bilingual children, Cambridge University Press.

JOHNSON, J., 2003, Language processing in bilingual children, Cambridge University Press.

MALAKOFF, M., HAKUTA, K., 2003, Language processing in bilingual children, Cambridge University Press.

MOČINIĆ, A., 2010, Bilinguismo e abilità metalinguistiche. Tesi di diploma, 
Università Juraj Dobrila di Pola.

SCOTTI JURIĆ, R., 2003, Bilinguismo precoce: funzioni e usi linguistici, EDIT, Fiume.

TITONE, R., 1993, Bilinguismo precoce ed educazione bilingue, Armando Editore, Roma.

\section{METALINGVISTIČKE SPOSOBNOSTI DVOJEZIČNIH I JEDNOJEZIČNIH UČENIKA}

$\mathrm{U}$ uvodnom dijelu rada prikazan je pregled istraživanja o kognitivnom funkcioniranju dvojezičnih osoba, kao i onih istraživanja koja navode prednosti i nedostatke dvojezičnosti. Istraživanja na području dvojezičnosti doista su brojna, no usprkos tome rezultati često ne dovode do jednoznačnih zaključaka. U cilju proširivanja prethodnih spoznaja o odnosu koji postoji između dvojezičnosti i metakognitivnog funkcioniranja, provedeno je istraživanje na 230 dvojezičnih i jednojezičnih učenika osnovnih škola. Dobiveni rezultati potvrdili su postojanje pozitivnih razvojnih efekata na metakognitivne sposobnosti, te značajno bolji učinak jednojezične djece u metajezičnim zadacima. Kao posebnu zanimljivost koja je razmatrana u raspravi navodimo činjenicu da su razvojne promjene mnogo veće kod dvojezične djece u odnosu na jednojezičnu.

Ključne riječi: dvojezičnost, metakognicija, djeca

\section{METALIGUISTIC ABILITIES IN BILIGUAL AND MONOLINGUAL PUPILS}

In the introduction of the current paper authors present a review of research regarding cognitive functioning of bilinguals, as well as reported advantages and disadvantages of bilingualism. Namely, the research in the area of bilingualism is rich, but the obtained results are often incongruent. With the purpose to clarify some knowledge regarding the existing relationship between bilingualism and metacognitive functioning, we conducted a research including 230 pupils, both bilingual and monolingual. Obtained results indicated positive effect of development for metacognitive abilities, and significant metalinguistic superiority of monolingual children. A very interesting finding is discussed further: developmental changes were more consistent for bilingual children.

Key words: bilingualism, metacognition, children 